\title{
Review of: "SITDP1 Is Required To Specify Tapetum Identity And For The Regulation Of Redox Homeostasis In Tomato Anthers"
}

\author{
Mohammed Alyemeni ${ }^{1}$
}

1 King Saud University

Potential competing interests: The author(s) declared that no potential competing interests exist.

Title: SITDPI IS REQUIRED TO SPECIFY TAPETUM IDENTITY AND FOR THE 2 REGULATION OF REDOX HOMEOSTASIS IN TOMATO ANTHERS

This manuscript, entitled “ SITDP1 IS REQUIRED TO SPECIFY TAPETUM IDENTITY AND FOR THE 2 REGULATION OF REDOX HOMEOSTASIS IN TOMATO ANTHERS is a good work done by the authors. This work can be accepted after minor revision.

Comments

1. Add some results (in percentage) in the abstract.

2. The introduction is not having latest citation. I suggest the authors should add latest citations.

3. Reactive oxygen species has been discussed in the manuscript but nothing has been provided in the introduction part. So the authors are suggested to write some para regrading the ROS.

4. Line 260-262: Peroxidases are multifunctional proteins that catalyze the oxidation of a variety of substrates by $\mathrm{H} 2 \mathrm{O} 2$ and act as efficient components of the antioxidative system controlling ROS (Ahmad et al., 2010,2019; Kohli et al., 2019; Mansoor et al., 2022). The sentence was without ref. I suggest the authors to add these refs in this statement.

- Ahmad P, Jaleel CA, Salem MA, Nabi G, Sharma S (2010) Roles of enzymatic and nonenzymatic antioxidants in plants during abiotic stress. Crit Rev Biotechnol. 30(3):161-75.

- Ahmad P, Tripathi DK, Deshmukh R, Singh VP, Corpas FJ (2019) Revisiting the role of ROS and RNS in plants under changing environment. Environ Exp Bot. 161: 1-3.

- Kohli SK, Khanna K, Bhardwaj R, Abde_Allaha EF, Ahmad P, Corpas FJ. (2019). Assessment of Subcellular ROS and NO Metabolism in Higher Plants: Multifunctional Signaling Molecules. Antioxidants.; 8(12):641.

- Mansoor S, Wani OA, Lone JK, Manhas S, Kour N, Alam P, Ahmad A, Ahmad P. (2022) Reactive Oxygen Species in Plants: From Source to Sink. Antioxidants, 11, 225.

Line 381-383: Interestingly, while high concentration of reactive oxygen species (ROS) cause irreversible DNA damage and cell death, at low levels ROS act as signalling molecules regulating cell division and cell 
fate (Kelliher \& Walbot, 2012; Yang et al., 2018; Ahmad et al., 2010,2019; Kohli et al., 2019; Mansoor et al., 2022). Add the above ref here in this statement.

Line 394-396: Enzymatic scavenging mechanism involve SOD, CAT and peroxidases, although peroxidases can act as both ROS generating and ROS-processing components (Mittler, 2017; Ahmad et al., 2010,2019; Kohli et al., 2019; Mansoor et al., 2022). Add the above ref here in this statement.

5. Result are not well written as I suggest the authors write the results in increase or decrease in percentage for all parameters. Authors have given comprehensive results but should be with percentage.

6. Discussion part is very week. The mechanism of action in the discussion part is not well documented. I suggest the authors should add mechanism of action, why and how a parameter is decreased or increased, what is the real mechanism?. It is important. Also authors should support their results by already published recent articles. 